\title{
A qui profite la concertation? Notes sur la concertation tirées de l'expérience lyonnaise, France
}

\section{Jean-Yves Toussaint, Sophie Vareilles, Lyon}

\section{Introduction}

A qui profite la concertation en aménagement urbain? L'intérêt porté par les auteurs à la concertation provient de commandes de recherches auxquelles l'équipe «Environnements \& Dispositifs Urbains» a répondu dans les années 2000 (Toussaint, VAREILles \& ZimmerMANN 2003; VAREILLES 2006). Ces commandes faisaient écho à des recherches sur l'expérience lyonnaise de requalification des espaces publics urbain portant sur la période 1990-2000 (Toussaint \& ZIMMERMANN 2001). Il s'agissait de comprendre les effets de la concertation sur les choix techniques en matière de requalification des espaces publics urbains. La concertation se présente aux publics urbains comme une double promesse, démocratique et technique. Elle promet une meilleure introduction des publics et de leurs usages dans le processus de décision affectant leurs environnements; elle promet aussi une ville plus conviviale, selon le sens donné par ILLICH (1973), comme relevant de la technique.

Les observations des pratiques lyonnaises de la concertation, qui constituent l'essentiel du corpus analysé (Encadré 1), montrent que les enjeux de la concertation sont moins centrés sur les partis techniques et urbains des aménagements que sur les configurations d'objets et de dispositifs techniques que le projet ordonne dans ces aménagements. Ainsi, la concertation doit contribuer à fonder la justesse de ces configurations en faisant valoir des intérêts sociaux et urbains. Ces observations ont conduit à penser que la concertation tend à subordonner les pratiques urbaines au processus de fabrication. Cette orientation participerait d'une tendance générale, dans la production d'objets et de dispositifs techniques, à subordonner les finalités d'usage à celle de la fabrication. En cela, la concertation ne tiendrait pas sa promesse.

L'article se propose de revenir sur cette double promesse de la concertation en trois points. Le premier consiste à mieux situer les enjeux techniques de la concertation à partir d'un cadre d'analyse sur la mobilisation des objets dans l'activité urbaine. Le deuxième présente l'expérience lyonnaise de la concertation et la promesse de concertation. Le troisième point intéresse les défauts de la concertation et la subordination de l'usage à la fabrication qu'elle contribue à maintenir.

\section{Objets et cités}

La massification des objets et des dispositifs techniques est une singularité de la société industrielle qui qualifie les villes actuelles. Ces villes n'ont plus de frontières et s'étalent au gré des dispositifs techniques et spatiaux qui les relient et finissent par former un milieu associé (Simondon 1989: 56-60), les environnements urbains. Ce milieu est formé par exemple de la voirie, des feux tricolores, des trottoirs, des arbres, des bancs, des automobiles, bref, d'un nombre fini d'objets dans la mesure où ces objets sont fabriqués, mais innombrables parce qu'impossibles à enfermer dans une liste exhaustive. La massification des objets a son pendant, celui des populations qui forment les urbains contemporains. Les objets sont mobilisés quotidiennement par une multitude d'individus. Une partie d'entre eux sert aux activités urbaines. Leurs assemblages produisent les environnements urbains qui autorisent la vie urbaine. Ce faisant, ils permettent à des milliards d'individus de s'affairer quotidiennement dans les zones urbanisées. Ils le font en accueillant la foule des objets que mobilisent ces individus. Ces objets sont appelés les «dispositifs techniques et spatiaux de l'urbain» (ToussaInT 2003; Toussaint \& ZimmermanN 2001). Généralement, ces dispositifs sont communs. Ils relèvent d'une forme de banalité et sont disponibles à tous. Leur existence relève de l'affectation d'une partie de l'impôt.

Les dispositifs techniques et spatiaux de l'urbain sont des instruments à la disposition des individus engagés dans l'activité sociale urbaine (ToussaINT 2009). Selon cette approche, ils sont appropriés aux activités sociales urbaines et comme instruments ils démultiplient les moyens d'agir pour chacun, individuellement ou collectivement. Cette approche instrumentale s'inspire de travaux en ergonomie cognitive (RABARDEL 1995) et d'une lecture de Simondon (1989). L'instrument est ici un outil de perception qui permet à celui qui en use de prélever de l'information sur le monde dans lequel il agit, de produire ainsi des connaissances utiles à la réalisation de ses desseins et d'être alors en situation d'invention dans ce monde. En ce sens l'instrument participe à l'autonomie de celui qui le mobilise dans ses activités. Cet effet de l'instrument renvoie aux positions développées par De CerTEAU (1980) sur la non-passivité des publics face aux objets fabriqués en leur nom. Dans cette perspective, tout aménagement est susceptible de délivrer de nouvelles licences d'action (Toussaint 2006; 2009) en appelant de nouveaux comportements individuels et collectifs. 


\section{Méthode d'observation}

L'analyse de l'expérience lyonnaise de la concertation reconstituée ici se rapporte, pour l'essentiel, à la politique de requalification des espaces publics urbains. L'observation et l'analyse de cette expérience s'appuient sur quatre dispositifs d'enquête:

- une revue d'articles de la presse locale se rapportant aux projets d'espaces publics urbains réalisés dans l'agglomération depuis 1989;

- une quarantaine d'entretiens menés auprès de praticiens (élus, fonctionnaires territoriaux, concepteurs, médiateurs) mobilisés dans les projets d'espaces publics;

- une observation participante réalisée dans le cadre d'un projet d'aménagement d'espace public urbain et dans le cadre de la rédaction de la Charte de la participation;

- cinq ateliers de discussion organisés entre 2000 et 2008 sur la pratique de la concertation rassemblant des élus, des chefs de projet, des concepteurs, des chargés de communication, des médiateurs, des représentations associatifs et des chercheurs.

Neuf études de cas ont été réalisées. Elles portent sur neuf projets de requalification d'espaces publics urbains dans l'agglomération lyonnaise. Ces projets, de 1990 à 2006, concernent différents types d'espaces publics urbains (hypercentre, centres des villes de la communauté urbaine, quartiers périphériques).

De cette façon, l'aménagement urbain produirait des offres en pratiques urbaines.

L'existence des dispositifs techniques et spatiaux de l'urbain comme instrument résulte de deux processus complémentaires formant la genèse instrumentale que sont l'instrumentation et l'instrumentalisation (RABARDEL 1995):

- L'instrumentation relève de l'activité de fabrication telle qu'elle assure l'existence des objets; elle intéresse la conception, la réalisation, la maintenance et l'obsolescence des objets fabriqués.

- L'instrumentalisation relève de la mobilisation des objets dans les activités sociales; elle intéresse le sens donné au fonctionnement des objets par les publics et les projections du public sur ce fonctionnement.

Les observations effectuées par GAULTIER (2007) suggèrent que tout objet fabriqué appelle des comportements individuels et collectifs qui varient selon leur disposition dans l'environnement des autres objets. Ces appels ou affordances (GiBson 1986) sont régulés collectivement par les usages. Chaque aménagement, en reconfigurant les dispositifs techniques et spatiaux de l'urbain, renouvelle l'offre en pratiques urbaines. Les licences d'action sont limitées par le discernement des publics. Celui-ci a partie liée avec les usages qui donnent sens au fonctionnement des dispositifs techniques et orientent les projections des publics. Ces règles d'usage s'imposent à tous comme modalités pour chacun d'user librement et singulièrement des dispositifs techniques et spatiaux disponibles à tous. Elles tendent aussi à tenir en l'état le fonctionnement des objets pour assurer la pérennité des services qu'ils rendent et des licences d'action qu'ils ouvrent. Ce rap- port des usages à la pérennité du fonctionnement est d'autant plus visible que les objets usés sont en partage et qu'ils sont usés simultanément par plusieurs individus. Les usages sont d'autant plus évidents et consubstantiels aux aménagements que les dispositifs techniques et spatiaux requis par les publics sont appropriés à leurs activités. De la qualité de cette appropriation dépend l'observance des règles d'usages. En réglant la mobilisation des dispositifs techniques et spatiaux de l'urbain, les usages règlent les pratiques, c'est-à-dire ce qui s'effectue hic et nunc pour chacun aux prises avec les autres et avec les objets nécessaires et mobilisés dans les cours d'action. Typiquement, marcher dans la rue implique des règles qui font que les activités de tous font sens pour chacun, que le comportement de chacun est escomptable par tous et que, sans échanger le moindre mot, ni coordonateur, tous cheminent. Ainsi, par la mise en commun et la banalité, les usages permettent de renoncer à la violence de l'appropriation (RAYmond 1988: 6). Ce faisant, ils réalisent, en l'incarnant dans les objets, la démocratie telle qu'en Europe elle se fonde dans les conjurations bourgeoises (Weber 1991) autour de la libre circulation des gens et des biens.

Finalement, les usages sont ce à partir de quoi s'évaluent à la fois le degré de convivialité des objets aux prises avec les urbains et le degré de civilité des urbains aux prises avec les objets - autrement dit, à partir de quoi s'évalue l'urbanité. En ce sens les dispositifs techniques et spatiaux de l'urbains procèdent de la convivialité (ILLICH 1973) et de la civilité (SchNAPPER 1994: 93-94) et concourent à l'autonomie des individus à la fois dans la ville et dans la cité. Bref, les dispositifs techniques et spatiaux sont ce par quoi la ville est habi- 
table. Ainsi, l'approche instrumentale permet de saisir le rapport aux objets qui constitue l'urbanité dans ces deux dimensions de la civilité et de la convivialité. Ce rapport aux objets s'entend avec une instrumentation qui répond aux usages qui lui préexistent.

L'observation des aménagements centrés sur la requalification des espaces publics urbains met en évidence le rapport qu'entretient le recours à la concertation avec les inquiétudes sur la capacité de l'aménagement à générer de l'urbanité. La question de la concertation tend à se poser avec le problème de l'instrumentalisation. Celui-ci émerge quand les objets sont produits hors des usages. Dans ces conditions, le sens donné aux fonctionnements des objets, les projections des publics sur ces fonctionnements et les pratiques urbaines ne sont plus régulés collectivement. L'instrumentalisation se trouve alors «séparée» de l'instrumentation. L'urbanité que recèlent la civilité et la convivialité est en germe ou en attente, elle apparaît comme un «spectre» (LEFEBVRE 1972: 102 et suivantes).

Les pratiques de concertation se développent toujours en contrepoint de dissentiments, voire de dissensions, sur les processus d'instrumentation et d'instrumentalisation. Elles apparaissent dans les années 1970 contre les rénovations urbaines des centres anciens promues par les édiles et les promoteurs et qui se traduisent par un éloignement des classes les plus populaires en banlieues. Elles reculent dans les années 1980 et subsistent dans les quartiers de grands ensembles; ceux-ci sont marqués par une urbanisation qui n'a pas généré d'urbanité et les publics urbains ont $\mathrm{pu}$ y fomenter des violences pour revendiquer leur appartenance à l'urbain (par exemple, au cours de l'été 1981, «Juillet noir» dans les Zones à Urbaniser en Priorité de Vénissieux, de Villeurbanne et de Vaulx-en-Velin, près de Lyon). Enfin, les pratiques de concertation réapparaissent à la fin des années 1990 à l'occasion d'un diagnostic de crise de la démocratie (RAGON 1977; VAREILLES 2006).

\section{La promesse de la concertation}

L'expérience lyonnaise de la concertation recouvre la période de développement de la concertation en France des années 1990 et 2000. Cette période se caractérise par une institutionnalisation des pratiques de concertation. Depuis 1990, huit lois ont resserré le cadre légal de ces pratiques (Tableau 1). Ce cadre règle l'interpellation des «publics» urbains par les «fabricants» de l'urbain. Par «fabricant», il faut entendre l'ensemble des rôles, des acteurs et des organisations qui sont chargés de la conception, de la réalisation, de la maintenance, du recyclage et de la destruction des dispositifs techniques et spatiaux de l'urbain. Cet ensemble regroupe par exemple les élus et les fonctionnaires des collectivités territoriales, les bureaux d'études techniques, les concepteurs, les entreprises de réalisation, celles de gestion. Par «publics» il faut entendre l'ensemble des individus qui dans leurs activités quotidiennes mobilisent ces dispositifs. Ils peuvent former des groupes ou des classes tels que des «habitants», des «riverains», de la «population», des «cyclistes», des «piétons», des «automobilistes», des «jeunes», des «commerçants».

L'expérience lyonnaise démarre avec la politique de requalification des espaces publics urbains en 1989 avec l'arrivée d'un nouveau maire à Lyon (ToussaINT \& Zimmermann 2001). Entre 1989 et 2000, une trentaine de dispositifs de concertation sont mis en œuvre (Tableau 1). L'agglomération de Lyon recourt à des dispositifs connus en France et en Europe (Centre D'ÉTUDES SUR LES RÉSEAUX, LES TRANSPORTS, L'URBANISME ET LES CONSTRUCTIONS PUBLIQUES - CERTU 2000; NeVeu 1999; VAReiLles 2006). Après les années 2000 , la concertation est systématique pour les aménagements urbains et intègre des chartes de «bonnes pratiques» (GRAND Lyon 2003). La diversité des pratiques mises en œuvre à Lyon met en évidence la polysémie du vocable «concertation». Ce fait n'est pas propre à Lyon. Il est général (BAcQue, Rey \& Sintomer 2005; Blanc 1988, 1995; Dion 1984; Leresche \& AudeBAT 2006; Mollet 1981). La «concertation» apparaît comme un «mot éponge» (Pacteau 1994). Comme une éponge, ce vocable s'enrichit des sens que lui attribuent ceux qui l'emploient et se plie à une multitude d'usages.

La concertation dans l'expérience lyonnaise est à l'initiative des personnels politiques et techniques des communes et de la communauté urbaine de Lyon (le Grand Lyon). Ces personnels trouvent des relais dans les associations, parmi les concepteurs (urbanistes et paysagistes) et dans le milieu de la recherche en sciences de l'homme et de la société. Le recours à la concertation se perpétue malgré les changements politiques dans les gouvernements de l'agglomération, en particulier au Grand Lyon. Cette collectivité territoriale en est l'acteur principal. Depuis 1989, plusieurs organisations de la concertation ont été testées en son sein. La responsabilité de la mise en œuvre de la concertation oscille longtemps entre le service de la communication et celui des espaces publics urbains. Bien que les premiers dispositifs de concertation aient été mis en œuvre en 1989 par une municipalité de droite, l'expérience lyonnaise de la concertation s'appuie sur les traditions des partis de gauche en la matière (notamment le socialisme municipal, voir Meuret 1982) et sur les expérimentations réalisées dans les années 1980 dans le cadre de l'application de la «politique de ville» dans les quartiers populaires (VoIsIN 2001) en réaction aux 


\begin{tabular}{|c|c|c|}
\hline Types & Sous-types & Dispositifs \\
\hline \multirow[t]{3}{*}{ Communication } & Les médias & $\begin{array}{l}\text { Journaux de chantier, panneaux de chantier, tracts, affiches, } \\
\text { sites Internet, porte-à-porte, journaux de quartier, bulletins } \\
\text { municipaux }\end{array}$ \\
\hline & Les expositions & L'exposition publique, le local d'information, la mission \\
\hline & Les manifestations festives & Fêtes, «journées habitantes», «activités pédagogiques» \\
\hline \multirow[t]{2}{*}{ Connaissance } & Les consultations & Sondages, questionnaires \\
\hline & Les enquêtes & $\begin{array}{l}\text { Enquêtes publiques*, études d'impact*, concertation } \\
\text { préalable*, enquêtes sociologiques }\end{array}$ \\
\hline \multirow[t]{3}{*}{ Implication } & Les réunions & $\begin{array}{l}\text { Réunions publiques, réunions de concertation, médiateur, } \\
\text { «habitants-relais» }\end{array}$ \\
\hline & Les groupes & Groupe de travail, atelier de concertation \\
\hline & Les conseils & $\begin{array}{l}\text { Comité d'initiative et de consultation d'arrondissement } \\
\text { (CICA)*, conseil de développement*, conseil de quartier*, } \\
\text { commissions extramunicipales et extracommunautaires }\end{array}$ \\
\hline
\end{tabular}

Tab. 1: Une typologie des dispositifs de concertation lyonnais, France

Les dispositifs suivis d'un astérisque $(*)$ sont des dispositifs légaux: la plupart sont obligatoires. L'enquête sociologique est un dispositif lyonnais qui consiste à établir les enjeux sociaux de l'espace public. Elle s'appuie sur des observations de l'espace public et sur des entretiens d'acteurs locaux et d'usagers.

Typologie der rechtlichen Partizipationsmöglichkeiten in Lyon, Frankreich

Typology of legal possibilities for participation in Lyon, France

violences urbaines de l'été 1981. Dans les années 2000, cette expérience s'adosse en partie aux préceptes du développement durable et se généralise à l'ensemble des politiques urbaines.

L'originalité de l'expérience lyonnaise de la concertation tient à sa primauté, à la diversité des dispositifs mis en œuvre et à leurs combinaisons. Les dispositifs de concertation peuvent être classés en trois grands types selon les rapports qu'ils permettent d'instaurer avec les publics: la communication aux publics, la connaissance des publics, l'implication des publics (Tableau 1). La communication informe les publics sur l'activité d'aménagement. Elle prévient des inconvénients du chantier et assure la promotion de l'urbanité projetée. La connaissance des publics sert à informer le projet d'aménagement des pratiques urbaines. Par exemple, l'enquête sociologique réalisée en phase d'études préalables permet de faire un état des pratiques existantes et de distinguer entre les «mauvais» usages, les «bons» usages, les usages absents et les usages disparus. Elle permet aussi d'identifier les demandes des publics urbains. L'implication met en scène l'activité de projet en reproduisant une partie des jeux de rôles internes au collectif d'énonciation (ToussaINT 1996) qu'institue le projet. Typiquement, les réunions publiques et les groupes de travail permettent, autour de l'exposition de plans d'aménagements, de faire valoir les enjeux de fabrication aux publics et d'évaluer les niveaux de satisfaction de ces publics pour l'aménagement urbain projeté.
Parmi la trentaine de dispositifs de concertation expérimentés depuis 1989, seule une partie a toujours cours. Les autres se sont avérés inadéquats aux buts opérationnels poursuivis ou en limites de légalité (VAREILLES 2006: 248-252). Ainsi en est-il du recours à l'expression littéraire d'écrivains censée révéler le potentiel d'usages des lieux (BEgAG et al. 1997). Trop ésotérique, ce type d'expression s'est révélé difficile à mettre en œuvre dans les projets. De même, les dispositifs permettant aux publics de s'exprimer sur les propositions des maîtres d'œuvre en votant avant la tenue du jury de concours s'avèrent contraires aux règles d'anonymat des concours en vigueur et tombent sous le coup de la loi. Finalement, l'enquête sociologique, les réunions publiques, les groupes de travail, les expositions, les tracts et les journaux de chantier, en raison de leur efficacité, forment l'essentiel des dispositifs de concertation utilisés dans les projets d'aménagement urbain.

La pratique de la concertation en aménagement urbain expérimentée au sein de l'agglomération lyonnaise lie les dimensions spatiale, technique, politique et sociale de l'urbanisation. Pour les élus, la concertation est l'occasion de rappeler publiquement les termes de leurs mandats tout en se soumettant au jugement de leurs mandants. Pour les élus et les fonctionnaires occupés à la question sociale, elle est un moyen de conforter les «liens sociaux»: elle vise à rapprocher les gouvernés des gouvernants en développant la prise de parole publique, l'écoute, la capacité de s'engager dans le 


\section{La promesse de démocratie urbaine}

«La démocratie locale a aujourd'hui profondément besoin de se nourrir de participation citoyenne pour répondre aux aspirations légitimes des habitants et faire face à la complexité croissante des politiques publiques. Ainsi la démocratie urbaine est à réinventer sur la base d'un dialogue renouvelé avec les usagers et les citoyens. Cela suppose entre autres d'ouvrir des espaces d'information, de concertation, de participation, pour construire une véritable dynamique avec les habitants» (GRAND LyON s.d.: 1).

«Des réunions publiques en atelier de concertation se jouent et se rejouent, en permanence, des temps d'information, d'écoute, de dialogue, de confrontation, de proposition et d'argumentation. Ces processus sont porteurs d'une plus grande intelligibilité de notre action, d'une meilleure efficacité de nos projets et d'une légitimité renouvelée de nos décisions. Tous les projets qui sont entrés en concertation en sont sortis différents, enrichis et mieux partagés. En ce sens ces démarches participatives constituent bien une aide à la décision pour les élus, une aide à la conception pour les équipes techniques, une aide à l'appropriation pour les habitants» (Grand Lyon 2005: 4).

projet et l'apprentissage de la citoyenneté. Pour tous, la concertation apparaît renouveler l'action publique en renforçant la légitimité des élus et des techniciens (Encadré 2). De ce renouvellement, il est attendu le recouvrement d'une forme d'urbanité fondée sur les «bons usages» des espaces aménagés. L'idée des promoteurs de la concertation est que, en informant les acteurs mobilisés dans le projet, la concertation contribue à une conception des aménagements plus facilement appropriables par les publics urbains, c'està-dire des aménagements urbains en adéquation avec les projections des publics, «plus adaptés à l'expression de la vie collective et aux pratiques des citadins» et permettant «de répondre plus justement aux attentes des usagers de la cité et des résidents» (VoIsin 2001: 147). Ainsi la concertation se justifie comme une promesse (Encadré 2). Cette promesse est double. Elle intéresse la démocratie représentative et le contrôle des mandataires par les mandants. Elle procède aussi à élargir aux publics la définition du cahier des charges et des programmes d'aménagement urbain. Dans cette double promesse, la concertation relève de l'instrumentation, elle participe des modalités par lesquelles les publics urbains sont dotés en objets techniques nécessaires à leurs activités quotidiennes.

\section{Les défauts de la concertation}

Les recherches conduites sur l'expérience lyonnaise de la concertation établissent la bonne foi des acteurs (fabricants et publics) engagés dans la concertation. Elles montrent aussi que la concertation augmente les interactions entre les fabricants et les publics et que l'augmentation de ces interactions est recherchée pour maximiser l'expression des publics à l'endroit des aménagements urbains. Mais l'accroissement de ces interactions ne semble pas permettre de tenir la pro- messe démocratique et technique de la concertation. La réalisation de cette promesse bute sur cinq grands travers de la concertation:

1) la tentation de l'acculturation des publics aux contraintes de l'instrumentation;

2) la tentation de l'expertise ès usages;

3) la tentation de la médiation;

4) la tentation de la surprogrammation des aménagements urbains;

5) la tentation de l'appropriation des aménagements par des publics.

Ces travers ne relèvent pas d'une stratégie globale des acteurs de la concertation, mais ils convergent pour limiter le processus d'instrumentalisation.

1) Le rapprochement des fabricants et des publics promu par la concertation apparaît ambigu. Il consiste en effet à acculturer les publics aux processus de fabrication (Grand Lyon 2003: 13; Grand Lyon 2005). Par exemple, des modules de formation à l'aménagement urbain sont intégrés aux ateliers et aux réunions des groupes de travail. Des activités pédagogiques organisées dans le cadre des écoles ou des centres sociaux sensibilisent les enfants à la maintenance des objets de nature (arbre, espaces verts) dans la ville. De manière générale, réunions, expositions, tracts et bulletins informent les publics sur le fonctionnement des dispositifs techniques et spatiaux de l'urbain et les instruisent sur les conditions d'énonciation et de projection de l'aménagement urbain (moyens disponibles, contraintes légales, régimes axiologiques dominants la fabrication). Cette acculturation vise à aligner les points de vue des publics sur ceux que les fabricants considèrent légitimes. Elle a pour effet d'altérer la capacité critique des publics à l'encontre de la fabrication en minorant les problèmes d'usage par rapport aux problèmes de fabrication. 
2) La concertation, en cherchant à intégrer l'opinion des publics dans les projets d'aménagement urbain, transforme les publics en experts ès usages (GRAND Lyon 2003: 10; VAREILles 2006: 218-225, 228-229). De cette manière les publics ne sont plus les mandants des fabricants, ils sont parties prenantes de la fabrication et endossent une partie de la responsabilité de l'aménagement. Autrement dit, les fabricants comme représentants des publics retournent à ceux-ci la responsabilité de l'effectuation de leur mandat, à savoir: construire, gérer et maintenir la ville et la cité. Ainsi, les publics perdent leur pouvoir critique et leur devoir de contrôle.

3) L'expression des publics recueillie par les dispositifs de concertation est difficilement exploitable et transposable dans les projets d'aménagement urbain. En dépit du recours à des objets intermédiaires (VINCK 1999) de plus en plus sophistiqués (entretiens, films, iconographies 3D, site Internet), la «parole du public» est l'objet d'interprétations aussi diverses que contradictoires (VAREILles 2006: 205-206). Pour être mise en œuvre dans le projet, l'expression des publics doit être affinée. Ce traitement se révèle d'autant plus nécessaire que l'expression des publics est sujette à caution. En effet, si les individus s'expriment en leur nom, ils ne représentent qu'eux-mêmes; s'ils parlent au nom d'un groupe (collectif, association, syndicat, parti politique), ils sont partisans. Cette fragilité de l'expression des publics interdit aux fabricants de reconnaître aux publics le moindre pouvoir prescriptif en matière d'aménagement (choix techniques, choix urbanistiques, choix urbains) (GRAND LyON 2003: 10). Ce pouvoir de prescription est transféré à des professionnels de la médiation.

Ces trois travers de la concertation tendent à limiter la capacité des publics non seulement à exprimer la demande en aménagement mais aussi à contrôler sur le plan de l'aménagement les mandats de ses représentants - y compris les mandats non électifs comme ceux octroyés par une expertise et la division du travail social (c'est-à-dire l'ensemble des acteurs mobilisés dans le projet et occupés à fabriquer la ville pour tous les autres, exceptés les élus). De cette manière, l'instrumentalisation est subordonnée à l'instrumentation dans le projet.

4) Le recours à la concertation est lié aux conflits d'appropriation. Pour les fabricants, ces conflits résultent d'appropriations inconvenantes. Cette inconvenance n'est pas évaluée du point de vue des groupes sociaux en conflit autour de l'urbanité, elle l'est à partir des normes de fabrication (notamment les normes de maintenance et de fonctionnement des dispositifs techniques et spatiaux ordonnés par les aménagements). La concertation permet de se garantir de ces conflits. En mettant en place un traitement de l'expression des publics, elle permet d'établir une correspondance entre des publics (ou groupes d'usagers) et des dispositifs techniques et spatiaux (AGENCE D'URBANISME DE LYON 1999; AgenCE D'URBANISME DE LyON \& GRAND LYON 1998). Cette correspondance permet aux fabricants de distribuer l'espace public urbain selon des catégories d'usages et des catégories de publics. Cette distribution sous-tend un découpage de l'espace urbain en aires spécialisées dans une activité urbaine et un type de public: par exemple, le terrain multisports et les «jeunes», le square et les «mères de famille et enfants», le terrain de boules et les «hommes». Cette distribution de l'espace est concomitante de la multiplication des dispositifs techniques et spatiaux spécialisés dans la ville. Ces dispositifs engagent les publics de manière explicite et redondante à agir selon la spécialité de chaque dispositif spécialisés. En cela, la concertation concourt à la «surprogrammation» de l'espace public urbain (Germain 2002). Ainsi, la concertation assure (en partie) le bon déroulement des programmes d'usage promus par les fabricants pour l'aménagement urbain. La question des usages glisse alors de la cité au projet.

5) L'appropriation par des publics apparaît comme un objectif de la concertation (CLAISSE 2007; GRAND LYON 2003; VoIsin 2001). Cette appropriation est l'affectation d'un espace à un public qui engage chaque public à faire «sien» les espaces que le projet d'aménagement lui destine. Elle est construite dans le projet autour d'une attente escomptée sur les comportements individuels et collectifs des publics attendus sur les espaces aménagés et appropriés. Dans cette forme d'appropriation, l'espace public urbain n'est plus en partage entre les publics, il est partagé selon des fonctions et des publics destinataires distincts. En ce sens cette forme d'appropriation fonde la surprogrammation.

Ce nouveau partage de l'espace public urbain modifie les modes d'institution des règles d'usage. Ces règles passent d'un régime d'appropriation aux activités des publics à un régime d'appropriation par des publics. Dans le premier régime, l'espace public urbain est commun à l'ensemble des publics et admet l'ensemble de ces publics selon des formes d'égards réciproques. Dans le second régime d'appropriation, l'espace public urbain est approprié par différents publics qui entendent y faire valoir des droits d'usage. Ainsi, dans le passage du premier régime au second, le devoir cède la place au droit. Ce changement de régime d'appropriation a pour effet le retournement de l'usage de la violence par la police urbaine. Celle-ci ne s'oppose plus à la violence de ceux qui s'approprient l'espace public urbain. Au contraire, elle a vocation à faire respecter le programme promu par les fabricants pour l'aménagement urbain: les propriétés programmées 
des dispositifs techniques et spatiaux et leur appropriation par les publics autorisés. Cette violence s'impose avec le recours d'instruments de communication, d'éducation et de contrôle des publics; par exemple les caméras de vidéosurveillance assurent la sûreté des espaces publics urbains en assurant le contrôle du bon déroulement des programmes d'usage.

Ces deux derniers travers tendent à nier la capacité d'invention des publics dans la ville et à donner l'exclusivité de la nouveauté aux fabricants, en particulier aux experts en innovation (architectes, paysagistes, artistes). Dans cette perspective, l'ensemble des travers de la concertation convergent pour subordonner l'instrumentalisation à l'instrumentation.

\section{Conclusion}

Cette analyse de la concertation à partir de l'expérience lyonnaise revient sur sa promesse d'une ville plus urbaine, à la fois plus conviviale et plus civile. Les observations et les résultats présentés recoupent ceux donnés par des expériences conduites dans les années 1970 et 1980 et par des expériences actuelles conduites dans d'autres villes françaises et européennes. Dans tous les cas, la concertation est une injonction adressée à la fois aux fabricants et aux publics urbains. Elle est à l'initiative des fabricants et recouvre des dispositifs et des pratiques très différentes. Elle doit permettre aux publics de comprendre les projets et d'adhérer à leur nécessité. En général, sa mise en œuvre soulève des difficultés et des questions quant à la représentation des publics urbains, leurs compétences à aménager la ville et la prise en compte de leur expression dans le projet (Bacque, Rey \& Sintomer 2005; Blanc 1995; CERTU 2000; Dion 1984; Helly 1999; Leresche \& Audebat 2006; Ragon 1977).

En cherchant à instaurer le dialogue avec les publics, les promoteurs de la concertation reconnaissent que la relation de la fabrication à l'usage est rompue. Cette reconnaissance renvoie au rapport qu'entretiennent les aménagements et les usages. Comme projet de fabrication, l'aménagement est aussi un projet social et politique. Les fabricants escomptent, à travers la configuration des dispositifs urbains constituant l'aménagement, des comportements individuels et collectifs des publics qui seront amenés à user de ces dispositifs dans leurs activités quotidiennes (ToussaINT 2003; 2006). L'analyse de l'expérience lyonnaise suggère que, dans la concertation, cette attente devient assignation, une assignation à des comportements définis et attendus dans leur adéquation aux fonctionnements des objets et dispositifs techniques que les aménagements ordonnent. Cette assignation correspond à une subordination de l'instrumentalisation à l'instrumentation.
Cette analyse, originale dans les études sur la concertation, peut s'appuyer sur une histoire de la concertation qui montre que celle-ci se développe contre les luttes urbaines et les conditions d'expression des publics urbains qu'elles ouvrent (AUTREMENT 1976; RAGON 1977; Tomas 1998). Elle peut aussi s'appuyer sur la surprogrammation de l'espace public urbain qui apparaît comme une donnée de l'aménagement urbain contemporain (Germain 2002; SCHMIDT \& Töllner 2009). Elle rejoint la position de Godbout (1987) pour qui la concertation est une tentative pour limiter la capacité d'opposition des gouvernés. Néanmoins, elle s'en éloigne lorsque GodBout attribue cette orientation de la concertation à la volonté des gouvernants de maximiser leurs avantages sur les gouvernés. La bonne foi observée des acteurs interrogés au cours des enquêtes menées engage à penser que cette orientation relèverait plutôt de la situation de fabrication et des exigences de production, c'est-à-dire de contraintes organisationnelles et économiques qui ne relèvent pas de la volonté d'individus ou de groupes.

Plus généralement, cette analyse de la concertation peut participer d'une critique de la fabrication. En cela, elle recoupe en partie la critique faite par ILLICH (1973). En reprenant les catégories de celui-ci, la subordination de l'instrumentalisation par l'instrumentation correspondrait au défaut de «convivialité» de l'hétéronomie de la productivité industrielle et du «monopole radical» des objets industriels. Ce défaut de convivialité limite l'appropriation des objets aux activités sociales au profit de leur appropriation par des groupes d'usagers. Cette dernière forme d'appropriation met à mal la civilité qu'implique la banalité des instruments de l'urbain. Sous des formes anémiées de la convivialité et de la civilité, l'urbanité ne peut s'effectuer et reste à l'état de virtualité.

Par ses travers, la concertation se révèle donc contreproductive du point de vue de l'urbanité. Elle ne tient pas sa promesse d'une meilleure démocratie et ne profite, en fin de compte, qu'à la fabrication.

\section{Bibliographie}

Agence D'urbanisme de Lyon (1999): Programmation. Evaluation des projets urbains en DSU. Fiches méthodologiques. - Lyon.

Agence d'urbanisme de Lyon \& Grand Lyon (1998): Méthode cartographique. Programmation et évaluation des projets urbains en DSU. Document de travail. - Lyon.

Autrement (1976): Contre-pouvoirs dans la ville. Enjeux politiques des luttes urbaines, Dossier 6. - Paris. Bacque, M.-H., Rey, H. \& Y. Sintomer (dirs) (2005): Gestion de proximité et démocratie participative. Une perspective comparative. - Paris: La Découverte. 
Begag, A., Bon, F., Cahuzac, P., Christin, P., Dextre, R., Doizelet, S., Drevet, P., Durif, M.B., Durif, E. Echenoz, J., Juliet, C., Laupin, P., Manet, E., Seysos, F., Sigaud, D. \& G. Walter (1997): Lyon ville écrite. Des lieux et des écrivains. - Paris: Stock.

Blanc, M. (1988): Concertation, sociologie urbaine, citoyenneté. - In: Les Annales de la Recherche Urbaine 38: 104-112.

Blanc, M. (1995): Politique de la ville et démocratie locale. La participation: une transaction la plus souvent différée. - In: Les Annales de la Recherche Urbaine 68-69: 98-106.

Centre D'Études SUR LES RÉSEAUX, LES TRANSPORTS, L'URBANISME ET LES CONSTRUCTIONS PUBLIQUES - CERTU (2000): La concertation en aménagement. Eléments méthodologiques. - Lyon: Collections «Aménagement et urbanisme» du CERTU.

Claisse, G. (2007): La communauté urbaine a progressé à tous les niveaux en matière de participation. - Lyon: Millénaire 3, Centre de Ressources Prospectives du Grand Lyon, http:/www.millenaire3.com/ Affichage-popup.128+M522b29a2583.0.html?\&tx_ ressm3_pi1[idunivers]=4 24.11.2009.

De Certeau, M. (1980): L'invention du quotidien. 1. Arts de faire. - Paris: Union générale d'édition.

Dion, S. (1984): Les politiques municipales de concertation. - In: Sociologie du travail 2: 121-140.

Gaultier, F. (2007): Comportements des usagers d'objets de l'espace public :études de bancs publics. - Lyon, Mémoire de master, Institut National des Sciences Appliquées de Lyon.

Germain, A. (2002): La découverte de l'espace public: regards d'architectes et de sociologues. - In: TomAs, F. (dir.): Espaces publics, architecture et urbanité de part et d'autre de l'Atlantique. - Saint-Etienne: Publications de l'Université de Saint-Etienne: 25-31.

GiBSon, J.J. (1986): The ecological approach to visual perception. - New Jersey: Hillsdale.

Godbout, J.T. (1987): La démocratie des usagers. - Montréal: Boréal Express.

Grand Lyon (2003): Charte de la participation. Plaquette. - Lyon.

GRAND LYON (2005): Charte de la participation. Rapport annuel 2004. - Lyon.

Grand Lyon (s.d.): Rapport annuel de la Commission consultative des services publics locaux 2003. - Lyon.

Helly, D. (1999): Une injonction: appartenir, participer. Le retour de la cohésion sociale et du bon citoyen. - In: Lien social et Politiques 41: 35-46.

ILLICH, I. (1973): La convivialité. - Paris: Seuil.

Lefebvre, H. (1972): Le droit à la ville, suivi de Espace et politique. - Paris: Anthropos.

Leresche, J.-P. \& M. Audebat (dirs) (2006): Participation et développement urbain durable. - In: Urbia - Les Cahiers du développement urbain durable 3, Lausanne.
Meuret,B.(1982):Le socialisme municipal.Villeurbanne 1880-1982. - Lyon: Presses Universitaires de Lyon.

Mollet, A. (dir.) (1981): Quand les habitants prennent la parole. - Paris: Plan Construction.

Neveu, C. (dir.) (1999): Espace public et engagement. Enjeux et logiques de la citoyenneté locale. - Paris: L'Harmattan.

Pacteau, C. (1994): L'évaluation aujourd'hui. Comment mesurer les compétences? - In: Sciences Humaines 35: 10-13.

RABARDEL, P. (1995): Les hommes et les technologies. Approche cognitive des instruments contemporains. - Paris: Armand Colin.

Ragon, M. (1977): L'Architecte, le Prince et la Démocratie. Vers une démocratisation de l'Architecture. - Paris: Albin Michel.

RAYMOND, H. (1988): Urbain, convivialité, culture. - In: Les Annales de la Recherche Urbaine 37:3-8.

SCHMIDT,A. \& M. TöLlNER (2009): La lumière urbaine en Allemagne - entre les besoins et les modes. - In: DeLEUIL, J.-M. (dir.): Eclairer la ville autrement. Innovations et expérimentations en éclairage public. - Lausanne: Presses Polytechniques et Universitaires Romandes: 235-255. SCHNAPPER, D. (1994): La communauté des citoyens. - Paris: Gallimard.

Simondon, G. (1989): Du mode d'existence des objets techniques. - Paris: Aubier.

Tomas, F. (1998): Vers une nouvelle culture de l'aménagement des villes. - In: ToussaInT, J.-Y. \& M. ZIMMERMANN (dirs): Projet urbain. Ménager les gens, aménager la ville. - Sprimont: Mardaga: 15-34.

Toussaint, J.-Y. (1996): Le collectif d'énonciation de l'espace: production contemporaine de l'espace et modalités de professionnalisation. - In: Espaces et Sociétés 84-85: 83-98.

Toussaint, J.-Y. (2003): Projets et usages urbains. Fabriquer et utiliser les dispositifs techniques et spatiaux de l'urbain. - Lyon: Habilitation à Diriger des Recherches, Université de Lyon II.

Toussaint, J.-Y. (2006): Le seuil. Un dispositif technique et spatial pour passer d'un monde à l'autre en employant son temps. - In: BAILlé, J. (dir.): Du mot au concept. Seuil. - Grenoble: Presses Universitaires de Grenoble: $139-157$.

Toussaint, J.-Y. (2009): Les usages et les techniques. In: STÉBÉ, J.-M. \& H. MARChAL (dirs): Traité sur la ville. - Paris: Presses Universitaires de France: 461-512.

Toussaint, J.-Y., VAreilles, S. \& M. ZimmermanN (2003): L'aménagement des espaces publics comme mise en œuvre de la démocratie. L'expérience lyonnaise de l'aménagement des espaces publics. Rapport final. Action «Projets urbains et nouvelles cultures urbaines». - Lyon: Atelier Cultures Urbaines.

Toussaint, J.-Y. \& M. ZimmermanN (dirs) (2001): User, observer, programmer et fabriquer l'espace public. - Lausanne: Presses Polytechniques et Universitaires Romandes. 
VAReILles, S. (2006): Les dispositifs de concertation des espaces publics lyonnais. Eléments pour une analyse du rôle de la concertation des publics urbains sur la fabrication de la ville. - Lyon: Thèse de doctorat, Institut National des Sciences Appliquées de Lyon.

VINCK, D. (1999): Les objets intermédiaires dans les réseaux de coopération scientifique. - Revue française de sociologie XL, 2: 385-412.

VoIsIn, B. (2001): Observer les lieux et les gens. Penser l'aménagement. - In: Toussaint, J.-Y. \& M. ZimmerMANN (dirs): User, observer, programmer et fabriquer l'espace public. - Lausanne: Presses Polytechniques et Universitaires Romandes: 147-156.

Weber, M. (1991): Histoire économique. Esquisse d'une histoire universelle de l'économie et de la société. - Paris: Gallimard.

\section{Résumé: A qui profite la concertation? Notes sur la concertation tirées de l'expérience lyonnaise, France} A qui profite la concertation en aménagement urbain? Aux fabricants et à la fabrication des aménagements urbains. En effet, l'observation de la concertation dans les projets de requalification des espaces publics urbains de l'agglomération lyonnaise (1989-2006) montre que la concertation tend à une tentative de subordination des usages à la fabrication. Cette observation met en défaut deux promesses de la fabrication contemporaine: celle d'une meilleure démocratie urbaine apportée par la concertation et celle de l'autonomie des publics urbains accrue par l'instrumentalisation des dispositifs techniques et spatiaux de l'urbain. La concertation est le fait des fabricants. Visant à l'efficacité de la fabrication, elle se produit aux dépens des usages. Ainsi, elle ne peut tenir les deux promesses et se révèle contre-productive du point de vue de l'urbanité et plus généralement de la civilité.

Mots-clés: concertation, aménagement urbain, civilité, urbanité, Lyon

\section{Summary: Who benefits from participation in urban planning? Comments on resident involvement in Lyon, France}

The article explores the motivation behind encouragement of resident participation in urban planning on the basis of experiences in Greater Lyon between 1989 and 2006. It suggests that participation is implemented to the benefit of the constructors, as it is they who benefit most. Although participation is promoted in contemporary civil construction in order to support greater urban democracy and increase urban public autonomy through the use of technical-spatial facilities, participation is also implemented in order to improve the performance of planned structures. Used for the latter reason, participation can not also increase public autonomy and democracy and can, in fact, be counterproductive for encouragement of urbanity and civility.

Keywords: participation, urban planning, civility, urbanity, Lyon

\section{Zusammenfassung: Wer profitiert von der Partizipa- tion? Anmerkungen zur Bürgerbeteiligung aus der Erfahrung von Lyon, Frankreich}

Wem nützt die Bürgerbeteiligung in der Stadtplanung? Sie nützt in erster Linie den Machern der städtischen Bebauung. Tatsächlich zeigen Ergebnisse aus den Bürgerbeteiligungsprojekten städtischer und öffentlicher Räume in der Agglomeration von Lyon (19892006) die Problematik zweier Zugeständnisse der zeitgenössischen Bebauung auf: dem einer gestärkten städtischen Demokratie durch die Bürgerbeteiligung und dem einer grösseren Autonomie der städtischen Öffentlichkeit durch die Nutzung technischer und räumlicher Anlagen. Bürgerbeteiligung muss von den unternehmerisch Tätigen ausgehen. Ist die Bürgerbeteiligung aber fast ausschliesslich auf die Leistungsfähigkeit der Infrastruktur ausgerichtet, dient sie nicht in erster Linie den Bürgern. In dieser Hinsicht kann Bürgerbeteiligung als Instrument die gemachten Versprechen nicht halten und wirkt bezüglich Urbanität und Zivilgesellschaft kontraproduktiv.

Schlüsselwörter: Bürgerbeteiligung, Stadtplanung, Zivilgesellschaft, Urbanität, Lyon

Prof. Dr. Jean-Yves Toussaint, Dr. Sophie Vareilles, Université de Lyon, INSA-Lyon, CNRS, UMR5600, F69364 Lyon, France.

e-mail:

jean-yves.toussaint@insa-lyon.fr

sophie.vareilles@insa-lyon.fr.

\section{Manuskripteingang/received/manuscrit entré le} 20.7.2009

Annahme zum Druck/accepted for publication/accepté pour l'impression: 5.1.2010 